\title{
A Randomized Clinical Trial of Metformin to Treat Autosomal Dominant Polycystic Kidney Disease
}

\author{
Stephen L. Seliger ${ }^{a} \quad K^{2}$ aleab Z. Abebe ${ }^{b}$ Kenneth R. Hallows ${ }^{c}$ Dana C. Miskulin ${ }^{d}$ \\ Ronald D. Perrone ${ }^{d}$ Terry Watnick $^{a}$ Kyongtae Tae Bae ${ }^{b}$ \\ a University of Maryland School of Medicine, Baltimore, MD, USA; ${ }^{b}$ University of Pittsburgh School of Medicine, \\ Pittsburgh, PA, USA; ' University of Southern California Keck School of Medicine, Los Angeles, CA, USA; ${ }^{\mathrm{d}}$ Tufts \\ Medical Center, Boston, MA, USA
}

\section{Keywords}

Autosomal dominant polycystic kidney · Disease ·

Metformin · Clinical trial

\begin{abstract}
Background: Metformin inhibits cyclic AMP generation and activates AMP-activated protein kinase (AMPK), which inhibits the cystic fibrosis transmembrane conductance regulator and Mammalian Target of Rapamycin pathways. Together these effects may reduce cyst growth in autosomal dominant polycystic kidney disease (ADPKD). Methods: A phase II, double-blinded randomized placebo-controlled trial of 26 months duration. Participants will include nondiabetic adults $(n=96)$ aged $18-60$ years, with an estimated glomerular filtration rate $(e G F R) \geq 50 \mathrm{~mL} / \mathrm{min} / 1.73 \mathrm{~m}^{2}$ and ADPKD, recruited from university-based practices in Baltimore and Boston. Participants will be randomized in 1:1 ratio to metformin or placebo at $500 \mathrm{mg}$ once daily, increased every 2 weeks to a maximum of $1,000 \mathrm{mg}$ twice daily as tolerated. Dose is decreased if eGFR falls to $30-45 \mathrm{~mL} / \mathrm{min} / 1.73 \mathrm{~m}^{2}$ and discontinued at eGFR $<30 \mathrm{~mL} / \mathrm{min} / 1.73 \mathrm{~m}^{2}$. Results: The primary outcomes are safety, assessed by the rates of hypoglycemia, elevated lactic acid levels, adverse events, and tolerability assessed by the Gastrointestinal Severity Rating Scale
\end{abstract}

and maximum tolerated dose of study medication. Secondary outcomes include changes in total kidney and liver volumes, pain, and health-related quality of life, and changes in urinary metabolomic biomarkers. Conclusions: Results of this trial will provide important information on the feasibility, safety, and tolerability of long-term use of metformin in patients with ADPKD and provide preliminary information regarding its efficacy in slowing disease progression. Furthermore, results may support or refute the hypothesis that metformin effects on disease progression are mediated through the activation of the AMPK pathway. These results will be essential for the justification and design of a full-scale efficacy trial.

๑) 2018 S. Karger AG, Basel

\section{Introduction}

Autosomal dominant polycystic kidney disease (ADPKD) is an autosomal dominant form of chronic kidney disease characterized by progressive enlargement of the kidneys by fluid-filled cysts resulting in kidney function decline. Fifty percent of affected individuals reach ESRD at a median of 50-60 years of age. Cystogenesis is believed to be central to the pathogenesis of kidney function deteriora-

\section{KARGER}

(c) 2018 S. Karger AG, Basel

E-Mail karger@karger.com

www.karger.com/ajn
Stephen L. Seliger, MD, MS

Department of Medicine, Division of Nephrology

University of Maryland School of Medicine

22 S Greene Street, Baltimore, MD 21201 (USA)

E-Mail sseliger@som.umaryland.edu 
tion, although only $5 \%$ of nephron segments contain cysts [1]. Other pathogenic mechanisms accounting for declining renal function include progressive inflammation and fibrosis in the non-cystic interstitium, which is believed to result from the release of inflammatory cytokines in response to ischemia from impingement of blood vessels by the enlarging cysts. Observational studies have shown strong correlations between the rate of total kidney volume growth (TKV) and estimated glomerular filtration rate (eGFR) decline and the time to reaching ESRD [2-4].

Growing evidence supports a therapeutic role of metformin in reducing cyst growth. Metformin may reduce cyst growth by inhibiting cyclic AMP generation in cells and via downstream effects of AMP-activated protein kinase (AMPK) activation in inhibiting the cystic fibrosis transmembrane conductance regulator (CFTR) and Mammalian Target of Rapamycin (mTOR) pathways [5]. Metformin-stimulated AMPK activation has been shown to phosphorylate and thereby inactivate the CFTR chloride channel $[6,7]$, reducing epithelial cell fluid secretion in ADPKD [8]. AMPK activation also directly and indirectly inhibits mTOR signaling via phosphorylation of mTORC1 and upstream phosphorylation of tuberous sclerosis complex 2 ( TSC2) [9], which in turn inhibits cell cycle progression and proliferation of cyst-lining cells. These biochemical changes as well as reduced fluid secretion and epithelial cell proliferation have been demonstrated in vitro. Furthermore, the administration of metformin in 2 different mouse models of ADPKD significantly reduced cystogenesis [10]. In addition to these preclinical findings, metformin has a favorable safety profile based on international experience with its widespread clinical use over nearly 60 years in the treatment of type 2 diabetes and polycystic ovarian disease. This makes metformin an attractive candidate for testing in ADPKD.

We have designed a phase II randomized placebo-controlled clinical trial to assess safety, tolerability, and effects of metformin treatment on kidney volume growth and eGFR in patients with early to moderate ADPKD (eGFR $\geq 50 \mathrm{~mL} / \mathrm{min} / 1.73 \mathrm{~m}^{2}$ ).

\section{Overview of Study Design}

The Trial for Administration of MEformin to tame PKD (TAME-PKD) trial is a multi-center phase II parallelgroup randomized double-blinded placebo-controlled trial to investigate the tolerability, safety, and efficacy of metformin in adults with ADPKD. This trial is funded by the US Department of Defense and registered with clinicaltri- als.gov (NCT02656017). The study is organized with 2 clinical centers (Tufts Medical Center, University of Maryland School of Medicine), a data coordinating center and an image analysis center (both located at the University of Pittsburgh School of Medicine), a metabolomics center (University of Southern California), and a Biorepository (University of Maryland). Potentially eligible patients with estimated GFR $\geq 50 \mathrm{~mL} / \mathrm{min} / 1.73 \mathrm{~m}^{2}$ will undergo a screening visit, which includes clinical and family history and laboratory testing. Those who meet eligibility criteria (see below) will be randomized in a 1:1 ratio to metformin (beginning at $500 \mathrm{mg}$ once daily) or matching placebo. A summary of study assessments and procedures is shown in Figure 1. Assessment of renal function, lactic acid, and other safety labs, along with symptoms and gastrointestinal (GI) tolerability, will occur every 2 weeks through 8 weeks, with upward titration of study medication at each interim visit if eGFR remains $\geq 45 \mathrm{~mL} / \mathrm{min} / 1.73 \mathrm{~m}^{2}$ and the drug is well tolerated, to a maximum dose of 1,000 mg twice daily. Participants will be evaluated in follow-up every 2 weeks through 8 weeks from randomization, then monthly until 24 weeks from randomization, and then every 3 months through 2 years from randomization. Two months after the completion of the 2-year follow-up period and termination of study drug, a close-out visit will occur. Abdominal magnetic resonance imaging (MRI) will be performed at baseline and every 6 months for quantification of TKV and total liver volume (TLV) as well as kidney cyst and liver cyst volumes. Urinary metabolites will be measured at baseline, after 1 and 3 months, then every 3 months through 2 years, and at the close-out visit. Enrollment commenced on June 27,2016, and is anticipated to conclude in June 2018, with the last follow-up in August 2020.

The primary outcomes of the study and their method of ascertainment are summarized in Table 1. These include medication tolerability as defined by: (a) GI symptom burden using the validated "Gastrointestinal Symptoms Rating Scale" (GSRS); (b) response to the question "Can you tolerate this dose of the study drug for the rest of your life?"; (c) maximally tolerated dose at 24 months; and (d) rates of study medication discontinuation. A co-primary outcome of safety will be defined by adverse events and serious adverse events, including prospectively defined and ascertained hypoglycemia and lactic acidosis. Secondary outcomes include: (a) ADPKD progression (height-adjusted TKV and eGFR decline); (b) medication adherence; (c) patient-reported pain and quality of life; (d) ESRD, death, and hospitalizations; and (e) changes in urinary metabolomics biomarkers including enzymes of the glycolytic pathway and markers of AMPK pathway activation. 


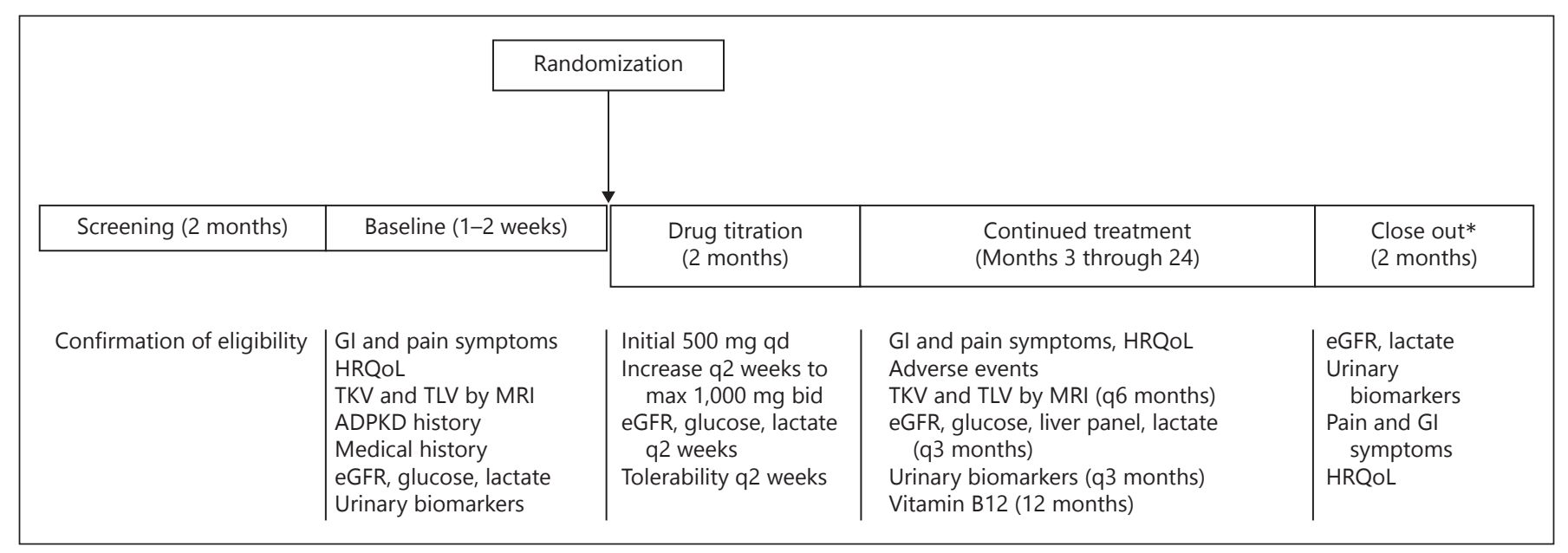

Fig. 1. Study procedures in each phase of TAME-PKD trial. * Two months after completion of the study drug.

\section{Eligibility}

Table 2 lists the eligibility criteria for participation. Overall, criteria were selected to exclude participants for whom metformin therapy would pose potential excess risk, or for whom randomization to placebo control would be inappropriate or infeasible. Eligible participants are nondiabetic adults aged $18-60$ years, with ADPKD as defined using the modified Pei-Ravine criteria [11]. There is no eligibility requirement based on TKV. Only patients with an eGFR of at least $50 \mathrm{~mL} / \mathrm{min} / 1.73 \mathrm{~m}^{2}$ are considered eligible due to the dependence of metformin on renal excretion and the increased incidence of adverse events including lactic acidosis in the setting of advanced renal disease [12]. Patients with vitamin B12 deficiency are also excluded unless the level can be corrected to the normal range, given the known effect of metformin in contributing to lower B12 levels.

\section{Dose Titration of Study Drug}

Consistent with the US Food and Drug Administration (FDA)-approved dosing guidelines for metformin, we selected a target dose of $1,000 \mathrm{mg}$ twice daily for use in this study. As most side effects of metformin are dose-dependent, a stepwise dose-titration protocol begins the study drug at $500 \mathrm{mg}$ once daily. Two weeks after starting medication, repeat safety labs including eGFR, glucose, and lactic acid are obtained, and patients are queried regarding their side effects with a focused GI symptoms questionnaire. If eGFR remains at least $45 \mathrm{~mL} / \mathrm{min} / 1.73 \mathrm{~m}^{2}$ without hypoglycemia or elevated lactic levels, and if the dose of study drug is deemed tolerable for long-term use by the participant, the dose is increased further to $500 \mathrm{mg}$ twice daily. The process is repeated at 4 and 6 weeks to a maximally tolerated dose of up to $1,000 \mathrm{mg}$ twice daily.

During follow-up, the study drug dose will be reduced to $500 \mathrm{mg}$ twice daily if eGFR is $<45 \mathrm{~mL} / \mathrm{min} / 1.73 \mathrm{~m}^{2}$ but $>30 \mathrm{~mL} / \mathrm{min} / 1.73 \mathrm{~m}^{2}$. If eGFR declines to $<30 \mathrm{~mL} /$ $\min / 1.73 \mathrm{~m}^{2}$, the study drug will be discontinued, until and if eGFR recovers to at least $30 \mathrm{~mL} / \mathrm{min} / 1.73 \mathrm{~m}^{2}$. Furthermore, eGFR will be monitored monthly rather than every 3 months if eGFR declines $<45 \mathrm{~mL} / \mathrm{min} / 1.73 \mathrm{~m}^{2}$. This dosing approach is motivated by revised FDA prescribing guidelines, which permit the use of metformin (with consideration of dose down-titration) in chronic kidney disease (CKD) patients whose eGFR is not $<30$ $\mathrm{mL} / \mathrm{min} / 1.73 \mathrm{~m}^{2}$ [13].

\section{Kidney and Liver Imaging}

The primary efficacy outcome of this phase II study will be the change in TKV and TLV adjusted for height (htTKV; htTLV). TKV is widely utilized as a surrogate measure of ADPKD severity in both observational and interventional studies, and is a strong prognostic marker for subsequent decline in GFR and progression to ESRD $[2-4,14]$. Abdominal MRI utilizing coronal T1and T2-weighted sequences without gadolinium contrast enhancement will be performed at baseline (prerandomization) and then repeated every 6 months at each clinical site. All MRI images will be reviewed and interpreted by the University of Pittsburgh image analysis center for computation of total kidney and TLVs as well as kidney cyst and liver cyst volumes. Volume estimates will be indexed to body height (i.e., height-adjusted TKV, htTKV). 
Table 1. Primary and secondary outcomes

\begin{tabular}{|c|c|}
\hline Outcome & Definition/ascertainment method \\
\hline \multicolumn{2}{|l|}{ Tolerability } \\
\hline GI symptoms & GSRS [15] - summary score \\
\hline Ability to tolerate medication & $\begin{array}{l}\text { Response to "Can you tolerate this dose of the study drug for the rest of } \\
\text { your life?" }\end{array}$ \\
\hline Medication discontinuation & Self-directed or physician-directed discontinuation of study medication \\
\hline Maximal tolerated dose of study medication & Highest daily dose during follow-up \\
\hline \multicolumn{2}{|l|}{ Safety } \\
\hline Hypoglycemia & Serum or plasma glucose $<60$, or $60-69 \mathrm{mg} / \mathrm{dL}$ with typical symptoms \\
\hline Adverse events & $\begin{array}{l}\text { Ascertained through questionnaire and communication with subjects at } \\
\text { each follow-up; categorized as serious or non-serious }\end{array}$ \\
\hline Lactic acidosis & $\begin{array}{l}\text { Clinically significant }(>5 \mathrm{mmol} / \mathrm{L}) \text { elevated blood lactate level, detected on } \\
\text { protocol-directed or clinically-directed laboratory test }\end{array}$ \\
\hline \multicolumn{2}{|l|}{ Secondary outcomes } \\
\hline TKV & Height-adjusted TKV by abdominal MRI \\
\hline TLV & Height-adjusted TLV by abdominal MRI \\
\hline Kidney function & Estimated GFR by CKD-Epi estimating equation \\
\hline HRQoL & SF-36 [19]: mental and physical component scores \\
\hline Pain & $\begin{array}{l}\text { Self-report back, radiating, and abdominal pain on HALT-PKD pain } \\
\text { questionnaire [20] }\end{array}$ \\
\hline Urinary metabolite concentrations (spot collection) & $\begin{array}{l}\text { Lactate, pyruvate, succinate, cAMP; standardized to urine creatinine } \\
\text { concentration }\end{array}$ \\
\hline AMPK pathway enzymes (spot urine) & $\begin{array}{l}\text { pAMPK-alpha and total AMPK-alpha, acetyl CoA carboxylase, and } \\
\text { ribosomal S6 kinase; standardized to urine creatinine }\end{array}$ \\
\hline Glycolytic pathway enzymes (spot urine) & $\begin{array}{l}\text { Lactate dehydrogenase A, pyruvate dehydrogenase kinase } 1 \text {, and pyruvate } \\
\text { kinase M2; standardized to urine creatinine }\end{array}$ \\
\hline
\end{tabular}

GI, gastrointestinal; GSRS, gastrointestinal safety rating scale; TKV, total kidney volume; TLV, total liver volume; HRQoL, health related quality of life; SF-36, short form-36; cAMP, cyclic AMP; AMPK, AMP-activated protein kinase; pAMPK, phosphorylated AMPK.

\section{Safety and Tolerability}

The most common side effects associated with metformin use are GI symptoms, which are typically dose-dependent. Accordingly, we will use the GSRS, a validated 15 -item questionnaire $[15,16]$ administered at baseline and during follow-up visits to quantify the severity of GI symptoms. The change in GSRS from baseline through follow-up will be compared between randomized groups. A second assessment of tolerability will be based on responses to the following question: "Can you tolerate this dose of the study drug for the rest of your life?" A negative response to this question will result in the down-titration of the study drug (see above). We will also compare the maximally tolerated dose of study medication and rates and reasons for study medication discontinuation between the metformin and placebo groups.

Hypoglycemia is an infrequent complication of metformin therapy in patient populations with or without diabetes. Under usual circumstances in diabetics, it does not occur in patients receiving metformin alone but could occur if caloric intake is deficient when strenuous exercise is not compensated by caloric supplementation or during alcohol intoxication. Patients will be monitored with serum glucose levels checked biweekly during study drug titration and every 3 months thereafter. While there are established values of fasting blood glucose levels for defining abnormal glucose tolerance, the lower limit of normal for fasting glucose in individuals without diabetes is not 
Table 2. Eligibility criteria

\begin{tabular}{|c|c|}
\hline Inclusion criteria & Rationale \\
\hline $\begin{array}{l}\text { Autosomal dominant polycystic kidney disease as } \\
\text { defined by unified pei-ravine criteria }\end{array}$ & $\begin{array}{l}\text { Standard clinical definition of this inherited renal disorder; } \\
\text { distinguishes ADPKD from acquired cystic renal disease }\end{array}$ \\
\hline Age $18-60$ years & $\begin{array}{l}\text { Exclude pediatric patients and those with advanced age but } \\
\text { only mild kidney function impairment. }\end{array}$ \\
\hline Fluent english-speaking & $\begin{array}{l}\text { Instructions and patient-reported outcomes are in } \\
\text { English language }\end{array}$ \\
\hline Able to provide written informed consent & $\begin{array}{l}\text { Must have cognitive capacity to provide informed } \\
\text { consent for participation }\end{array}$ \\
\hline Exclusion criterion & Rationale \\
\hline $\begin{array}{l}\text { Estimated GFR }<50 \mathrm{~mL} / \mathrm{min} / 1.73 \mathrm{~m}^{2} \text { (estimated from serum } \\
\text { creatinine using the } \mathrm{CKD} \text {-epi equation) }\end{array}$ & $\begin{array}{l}\text { Excess risk of metformin adverse drug reactions including } \\
\text { lactic acidosis with more advanced renal impairment }\end{array}$ \\
\hline $\begin{array}{l}\text { Diabetes (currently diagnosed, or fasting glucose }>126 \mathrm{mg} / \mathrm{dL} \text {, } \\
\text { or non-fasting glucose }>200 \text { ), or HbA1C }>6.5\end{array}$ & $\begin{array}{l}\text { Patients may require metformin or other oral hypoglycemic } \\
\text { agent for clinical care }\end{array}$ \\
\hline $\begin{array}{l}\text { Pregnancy or lactation or intending to become pregnant } \\
\text { within the next } 3 \text { years }\end{array}$ & Risk of metformin to fetus/infant \\
\hline Unstable or unclipped ( $>7 \mathrm{~mm}$ ) cerebral aneurysm & High risk of acute intracranial hemorrhage, which is often fatal \\
\hline $\begin{array}{l}\text { Implanted ferromagnetic object (e.g., pacemaker) or } \\
\text { severe claustrophobia }\end{array}$ & Contraindication to MRI \\
\hline $\begin{array}{l}\text { Systemic disease (other than hypertension) likely to } \\
\text { contribute to kidney disease (e.g., lupus) }\end{array}$ & Exclude patients with renal diseases other than ADPKD \\
\hline Uncorrectable vitamin B12 deficiency & $\begin{array}{l}\text { Metformin may reduce B12 levels leading to worsening } \\
\text { deficiency }\end{array}$ \\
\hline $\begin{array}{l}\text { Active coronary artery disease, which will be defined } \\
\text { as presence of stable or unstable angina }\end{array}$ & $\begin{array}{l}\text { Exclude patients expected to suffer acute cardiovascular events } \\
\text { during } 2 \text {-year follow-up }\end{array}$ \\
\hline $\begin{array}{l}\text { Use of medications that may interact with metformin: } \\
\text { Nifedipine } \\
\text { Furosemide } \\
\text { Cationic drugs (amiloride, ranitidine, triamterene digoxin, } \\
\text { procainamide, quinidine, vancomycin, trimethoprim) }\end{array}$ & $\begin{array}{l}\text { Minimize drug-drug interactions that can increase potential } \\
\text { toxicity of metformin }\end{array}$ \\
\hline Participation in a clinical trial with study medications & $\begin{array}{l}\text { Potential interaction between metformin and investigational } \\
\text { drugs on primary and secondary outcomes }\end{array}$ \\
\hline Active military personnel & $\begin{array}{l}\text { DoD regulations regarding enhanced protections of active } \\
\text { duty military personnel }\end{array}$ \\
\hline Any solid organ transplant & Hypothesis relates to progression of native ADPKD \\
\hline History of non-compliance & Exclude patients unlikely to comply with study protocol \\
\hline Allergy or intolerance to metformin & Safety \\
\hline Solitary kidney & TKV (primary efficacy outcome) would be invalid \\
\hline
\end{tabular}

ADPKD, autosomal dominant polycystic kidney disease; TKV, total kidney volume; MRI, Magnetic Resonance Imaging; HbA1C, Hemoglobin A1C. 
as clear $[17,18]$. Hypoglycemia is defined as a blood glucose level $<70 \mathrm{mg} / \mathrm{dL}$ if it is accompanied by typical symptoms and signs of hypoglycemia (e.g., shakiness, tachycardia, diaphoresis), or blood glucose $<60 \mathrm{mg} / \mathrm{dL}$ with or without symptoms. In the event of hypoglycemia, study medication will be down-titrated (if a mild event) or discontinued (if severe). Lactic acidosis is a rare but lifethreatening complication of metformin use, whose incidence is greater with lower eGFR. Venous lactate levels will be measured at baseline and during follow-up. Episodes of significantly elevated lactic acid (e.g., $>5 \mathrm{mmol} / \mathrm{L}$ ) will result in emergency care as appropriate, and immediate termination of study medication. Risk of lactic acidosis will be mitigated by careful dose titration with lower eGFR. Further, patients will be carefully counseled to temporarily hold their study medication prior to surgery or iodinated contrast exposure, and during episodes of AKI. Other adverse events will be collected and categorized by severity and type according to the NIH-sponsored Common Terminology Criteria for Adverse Events version 4.0 and compared between metformin and placebo groups.

\section{Health Related Quality of Life}

Metformin is hypothesized to reduce TKV growth, which may result in reduced pain and other symptoms relating to organ enlargement. Health related quality of life will be quantified by the Medical Outcomes Study Short Form-36 [19], and pain will be quantified by the HALT-PKD Pain Questionnaire, a 27-item instrument encompassing pain location, severity, frequency, and interference with daily functions [20].

\section{Metabolic Analysis}

Concentrations of targeted metabolites and enzymes of the glycolytic and AMPK pathways will be quantified in urine at baseline and during follow-up to test whether these markers may correlate with disease severity, progression, and potential response to metformin therapy. Of note, recent work suggests that there is dysregulated cellular metabolism in ADPKD tissue, with excessive reliance on aerobic glycolysis, the so-called Warburg effect [21]. Metabolites (lactate, pyruvate, succinate, cyclic AMP, and creatinine) will be quantified by enzymatic assays. Key glycolytic pathway enzymes (lactate dehydrogenase $\mathrm{A}$, pyruvate dehydrogenase kinase 1 , and pyruvate kinase M2), previously found to be elevated in ADPKD mouse and patient samples [21], will be quantified by enzyme-linked immunosorbent assays. Enzymes that reflect AMPK pathway activity (phosphorylated AMPK-alpha and total AMPK-alpha, phosphorylated acetyl CoA

A Randomized Clinical Trial of

Metformin to Treat ADPKD carboxylase and total acetyl CoA carboxylase, and phosphorylated ribosomal P70 S6 kinase [pP70S6K]) will be quantified by Western blotting. Analyte levels will be normalized to urinary creatinine concentrations.

Additional blood and urine samples collected at study visits will be stored in a biorepository, with samples available to qualified investigators after completion of the clinical trial.

\section{Data and Safety Monitoring}

An independent Data and Safety Monitoring Board (DSMB) with expertise in ADPKD and diabetes has been chartered by the Steering Committee. The DSMB will meet every 3 months until the first 10 enrolled participants have completed 3 months of follow-up, and then every 6 months thereafter. Consistent with the phase II design of this study, there will be no planned interim analysis of efficacy as the study is not powered for efficacy. The DSMB will report to the sponsor (Department of Defense) and the Steering Committee. An independent Research Monitor with expertise in diabetes treatment will also be responsible for monitoring and coordinating all patient safety measures, including measures in response to adverse events potentially related to metformin. Both the Research Monitor and the DSMB have authority to stop the research protocol for safety concerns.

\section{Statistical Analysis and Sample Size Estimation}

The full analysis set will be based on an intention-totreat analysis, comprising all participants randomized to either study arm. A sensitivity per-protocol analysis will also be performed including participants who have successfully completed drug titration with a maximum tolerated dose. The safety analysis set will be a subset of the intention-to-treat set and include participants who have at least received and taken metformin or placebo. The primary outcome of GI tolerability will be compared between randomized groups using linear mixed models, with the GSRS score as the primary dependent variable, with a random intercept to allow for participant-level variability of baseline GSRS. Testing for time-by-study arm interaction will determine whether the change in GSRS scores differ between groups. Medication adherence (defined as $\geq 80 \%$ of prescribed pills taken) will be compared using the chi-square test, and the log-rank test used to compare time to medication discontinuation. For safety, we will calculate cumulative incidence between study arms and use logistic regression to assess the association of study arms with the proportion of participants affected across various AEs and SAEs. 
For the secondary outcomes, a mixed linear regression model per Laird and Ware [22] will be fit with the natural $\log$ of htTKV (lnhtTKV) as a function of time, the interaction between time and study arm, and clinical site. To account for the participant-level variabilities of baseline $\operatorname{lnTKV}$ as well as rate of change, the model intercept and slope will be allowed to vary if needed. A significant interaction between time and study arm could indicate a slowing of PKD progression due to metformin. Estimated GFR will be compared between metformin and placebo using a similar Laird and Ware [22] model as above. Urinary biomarker concentrations (standardized to creatinine concentration) as a function of follow-up time will also be compared between metformin and placebo groups using linear mixed models. We will also estimate crosssectional and longitudinal effects of these biomarkers on TKV and eGFR, to determine their predictive importance in $\mathrm{ADPKD}$ progression. For these predictive analyses, we will utilize linear mixed models incorporating the following predictors: time, time-by-study arm interaction, baseline biomarker of interest, time-by-biomarker interaction, and the 3-way interaction of these predictors. These models may identify subgroups of participants who might or might not benefit from metformin therapy, although such subgroup analyses will be considered exploratory and may not have sufficient statistical power.

Sample size estimations for the primary outcomes focus on the $95 \%$ CI width for relevant point estimates of each outcome within each study arm. With 48 participants per study arm and $15 \%$ attrition (similar to the TEMPO trial) [23], we will have the ability to estimate a CI width no larger than 21 for the primary tolerability outcome of GSRS score, and a CI width no larger than 0.25 for medication discontinuation.

\section{Discussion}

This phase II randomized controlled trial will provide measures of the tolerability, safety, and efficacy of metformin in adult patients with ADPKD and preserved or mildly decreased kidney function. This information will be instrumental in supporting the design of a larger, longterm multicenter phase III efficacy trial.

Although preclinical studies have demonstrated that metformin can substantially slow cyst progression in rodent models of ADPKD, potentially mediated through the activation of AMPK, the effect of this agent on disease progression in human patients has not been studied. Furthermore, although the overall safety and tolerability of metformin has been demonstrated in non-ADPKD patients (with type 2 diabetes, with obesity and impaired glucose tolerance, and with polycystic ovarian syndrome), there are potential safety and tolerability challenges with the use of metformin in ADPKD. First, metformin is largely excreted by the kidneys, such that drug half-life is substantially prolonged when GFR is reduced. The risk of serious adverse drug effects such as lactic acidosis is increased in patients with reduced GFR, although there is debate regarding the magnitude of this excess risk, with more recent studies suggesting the risk is very modest in those with eGFR $>30 \mathrm{~mL} / \mathrm{min} / 1.73 \mathrm{~m}^{2}$ and without acute kidney injury [13]. Initial FDA-approved prescribing guidelines advised against the use of metformin in men with serum creatinine $>1.5 \mathrm{mg} / \mathrm{dL}$ and women with serum creatinine $>1.3 \mathrm{mg} / \mathrm{dL}$. However, in response to extensive data supporting the safety of metformin in CKD patients without severely reduced GFR, the FDA revised these guidelines in April 2016 [13]. These revised guidelines recommend that metformin can be initiated safely in patients with eGFR as low as $45 \mathrm{~mL} / \mathrm{min} / 1.73 \mathrm{~m}^{2}$ and can be continued after initiation provided eGFR remains at least $30 \mathrm{~mL} / \mathrm{min} / 1.73 \mathrm{~m}^{2}$. The revised FDA guidelines are similar to those promulgated by the Endocrine Society [24].

The revised guidelines motivate the eligibility criteria and dose-titration procedures in TAME-PKD. Specifically, frequent monitoring of eGFR will occur during the first 6 months, with down-titration and/or holding of study drug based if eGFR declines as described above. Although cases of lactic acidosis in metformin-treated patients are most commonly highly symptomatic diseases associated with critical illness [25], this study protocol measures venous lactate levels during routine follow-up, with the rationale that subclinical lactic acidosis may precede clinically overt disease. A comparison of on-treatment lactic acid levels between the metformin and placebo groups may also demonstrate whether metformin increases serum lactate levels in ADPKD patients. Of note, a prior study in type 2 diabetics with average serum creatinine $1.6 \mathrm{mg} / \mathrm{dL}$ did not find greater average lactate levels in metformin-treated patients as compared to control subjects, although lower renal function correlated with higher lactate levels regardless of treatment [26].

GI symptoms (e.g., nausea, dyspepsia, and diarrhea) are among the most common side effects observed in patients taking metformin and are typically dose-dependent. Such side effects could plausibly be more frequent in patients with $\mathrm{ADPKD}$, who may be more prone to GI symptoms related to progressive kidney and liver enlargement. We therefore focused on quantifying GI 
symptoms as a primary measure of tolerability, utilizing a validated patient-reported questionnaire $[15,16]$. The GSRS instrument has been implemented in studies of primary GI disorders and has been utilized to quantify medication side effects due to different preparations of mycophenolate in renal transplant recipients [27]. Demonstrating long-term tolerability of metformin in ADPKD patients is an essential prerequisite to justifying a longerterm phase III clinical efficacy trial. High rates of medication intolerability leading to drug discontinuation have been observed in clinical trials of other pharmacotherapies in ADPKD [23, 28, 29].

TAME-PKD is not designed to be able to detect clinically meaningful differences between metformin and placebo groups in the rate of kidney functional decline and ESRD. However, this trial will provide preliminary estimates of the effect of metformin compared to placebo on change in TKV as assessed by serial MRI studies. TKV is a widely utilized measure of ADPKD severity, especially among patients with more preserved GFR [2-4, 14]. Change in TKV has been considered a surrogate disease outcome in such ADPKD patients in studies of other pharmacological interventions [23, 28, 29]. Furthermore, the FDA has accepted TKV as a valid prognostic marker for the long-term risk of renal function decline in ADP$\mathrm{KD}$ [30]. The recently published tolvaptan trials showed a significant reduction in the rate of TKV growth [23] as well as in eGFR decline with treatment with a V2 receptor antagonist [31].

The TAME-PKD trial is also designed to address important mechanistic questions regarding the effects of metformin on enzymes and metabolites of the glycolytic pathway, along with AMPK pathway enzymes, and how such effects influence subsequent disease progression. Prior preclinical studies have suggested that AMPK activation in renal tubular cells inhibits both mTOR (thereby inhibiting cell grown and proliferation) and CFTR (inhibiting fluid secretion). In kidney cells, a PKD1 mutation alters energy metabolism and oxygen sensing by inducing aerobic glycolysis and inhibiting oxidative metabolism, which in turn has the deleterious effect of inhibiting AMPK activity [21]. This trial will test the hypothesis in human patients that treatment with the AMPK activator metformin in ADPKD will alter urinary metabolomic and AMPK pathway biomarkers. It will further explore whether such biomarkers may correlate with disease severity, prognosis, and response to metformin treatment.

In conclusion, the TAME-PKD trial will for the first time test the safety, tolerability, and efficacy of metformin compared to placebo in patients with ADPKD. It will also test the effects of metformin on metabolomic biomarkers, and examine whether such effects predict clinical response in terms of disease progression. Results of this trial will be instrumental in supporting the design of a phase III clinical efficacy trial, and will further provide important data on the mechanisms of disease progression.

\section{Acknowledgment}

The authors wish to thank the following research staff for their tireless efforts in the conduct of this clinical trial: Carly Tucker and Margaret Healey (Tufts Medical Center), Charalett Diggs and Pushpawallie Dhilipkannah (University of Maryland), Linda Whiting, Sharon Stover and Cheng Tao (University of Pittsburgh).

\section{Financial Disclosure}

R.D.P. serves as consultant to Otsuka, Regulus, Sanofi, Vertex, PalladioBio, receives research support from Otsuka and Kadmon Corporation, and is section editor for the Cystic Disease section for Uptodate. S.L.S. receives research support from Otsuka and Kadmon Corporation. T.W. has approved and pending patents relating to DNA testing in ADPKD. She serves on the Board of Trustees and Scientific Advisory Committee of the PKD Foundation. She receives research support from Ostuka and Kadmon Corporation. K.R.H. serves on a medical advisory board for Otsuka. K.T.B. receives research support from Kadmon Corporation.

\section{Support}

The clinical trial is supported by a grant from the Department of Defense Congressionally Directed Medical Research Program (W81XWH-15-1-0663, PI: Bae). A grant from the NIDDK (P30DK090868) supports Drs. Stephen L. Seliger and Terry Watnick and the University of Maryland site, with additional support by the University of Maryland Institute for Clinical and Translational Research and the University of Maryland General Clinical Research Center. Dr. Kenneth R. Hallows is also supported by another grant from the Department of Defense (W81XWH-15-1-0420). A grant from the National Center for Advancing Translational Sciences (UL1 TR001064) supports the Tufts Medical Center site and Dr. Ronald D. Perrone. Biorespository storage is supported by the University of Maryland School of Medicine Center for Innovative Biomedical Resources, Biorepository, Baltimore, Maryland.

\section{Author Information}

All authors contributed to the research idea and study design of the TAME-PKD trial. The manuscript was initially drafted by S.L.S and D.C.M. and critically revised by K.Z.A., K.T.B., K.R.H., R.D.P., and T.W. Each author contributed important intellectual content and accepts accountability for the overall work. 


\section{References}

1 Grantham JJ, Geiser JL, Evan AP: Cyst formation and growth in autosomal dominant polycystic kidney disease. Kidney Int 1987;31: 1145-1152.

-2 Chapman AB, Bost JE, Torres VE, GuayWoodford L, Bae KT, Landsittel D, Li J, King BF, Martin D, Wetzel LH, Lockhart ME, Harris PC, Moxey-Mims M, Flessner M, Bennett WM, Grantham JJ: Kidney volume and functional outcomes in autosomal dominant polycystic kidney disease. Clin J Am Soc Nephrol 2012;7:479-486.

-3 Irazabal MV, Rangel LJ, Bergstralh EJ, Osborn SL, Harmon AJ, Sundsbak JL, Bae KT, Chapman AB, Grantham JJ, Mrug M, Hogan MC, El-Zoghby ZM, Harris PC, Erickson BJ, King BF, Torres VE; CRISP Investigators: Imaging classification of autosomal dominant polycystic kidney disease: a simple model for selecting patients for clinical trials. J Am Soc Nephrol 2015;26:160-172.

4 Perrone RD, Mouksassi MS, Romero K, Czerwiec FS, Chapman AB, Gitomer BY, Torres VE, Miskulin DC, Broadbent S, Marier JF: Total kidney volume is a prognostic biomarker of renal function decline and progression to end-stage renal disease in patients with autosomal dominant polycystic kidney disease. Kidney Int Rep 2017;2:442-450.

$\checkmark 5$ Miller RA, Chu Q, Xie J, Foretz M, Viollet B, Birnbaum MJ: Biguanides suppress hepatic glucagon signalling by decreasing production of cyclic AMP. Nature 2013;494:256.

6 Hallows KR, Kobinger GP, Wilson JM, Witters LA, Foskett JK: Physiological modulation of CFTR activity by AMP-activated protein kinase in polarized T84 cells. Am J Physiol Cell Physiol 2003;284:C1297-C1308.

7 Hallows KR, Raghuram V, Kemp BE, Witters LA, Foskett JK: Inhibition of cystic fibrosis transmembrane conductance regulator by novel interaction with the metabolic sensor AMP-activated protein kinase. J Clin Invest 2000;105:1711-1721

$>8$ Davidow CJ, Maser RL, Rome LA, Calvet JP, Grantham JJ: The cystic fibrosis transmembrane conductance regulator mediates transepithelial fluid secretion by human autosomal dominant polycystic kidney disease epithelium in vitro. Kidney Int 1996;50:208-218.

$>9$ Dibble CC, Cantley LC: Regulation of mTORC1 by PI3K signaling. Trends Cell Biol 2015;25:545-555.

-10 Takiar V, Nishio S, Seo-Mayer P, King JD Jr, Li H, Zhang L, Karihaloo A, Hallows KR,
Somlo S, Caplan MJ: Activating AMP-activated protein kinase (AMPK) slows renal cystogenesis. Proc Natl Acad Sci U S A 2011;108: 2462-2467.

11 Pei Y, Obaji J, Dupuis A, Paterson AD, Magistroni R, Dicks E, Parfrey P, Cramer B, Coto E, Torra R, San Millan JL, Gibson R, Breuning M, Peters D, Ravine D: Unified criteria for ultrasonographic diagnosis of ADPKD. J Am Soc Nephrol 2009;20:205-212.

12 Bailey CJ, Turner RC: Metformin. N Engl J Med 1996;334:574-579.

13 Administration FaD: FDA Revises Warnings Regarding Use of the Diabetes Medicine Metformin in Certain Patients with Reduced Kidney Function; in Administration UFaD (ed), 2016.

14 Alam A, Dahl NK, Lipschutz JH, Rossetti S, Smith P, Sapir D, Weinstein J, McFarlane P, Bichet DG: Total kidney volume in autosomal dominant polycystic kidney disease: a biomarker of disease progression and therapeutic efficacy. Am J Kidney Dis 2015;66:564576.

15 Svedlund J, Sjodin I, Dotevall G: GSRS - a clinical rating scale for gastrointestinal symp toms in patients with irritable bowel syndrome and peptic ulcer disease. Dig Dis Sci 1988;33:129-134.

16 Revicki DA, Wood M, Wiklund I, Crawley J: Reliability and validity of the Gastrointestinal Symptom Rating Scale in patients with gastroesophageal reflux disease. Qual Life Res 1998;7:75-83.

17 Mitrakou A, Ryan C, Veneman T, Mokan M, Jenssen T, Kiss I, Durrant J, Cryer P, Gerich J: Hierarchy of glycemic thresholds for counterregulatory hormone secretion, symptoms, and cerebral dysfunction. Am J Physiol 1991; 260(1 pt 1):E67-E74.

18 Service FJ: Hypoglycemic disorders. N Engl J Med 1995;332:1144-1152.

19 Ware JW, Kosinski M, Gandek B: SF-36 Health Survey: Manual and Interpretation Guide. Lincoln, Quality Metric Inc, 2002.

20 Bajwa ZH, Sial KA, Malik AB, Steinman TI: Pain patterns in patients with polycystic kidney disease. Kidney Int 2004;66:15611569.

21 Rowe I, Chiaravalli M, Mannella V, Ulisse V, Quilici G, Pema M, Song XW, Xu H, Mari S, Qian F, Pei Y, Musco G, Boletta A: Defective glucose metabolism in polycystic kidney disease identifies a new therapeutic strategy. Nat Med 2013;19:488-493.
22 Laird NM, Ware JH: Random-effects models for longitudinal data. Biometrics 1982;38: 963-974.

23 Torres VE, Chapman AB, Devuyst O, Gansevoort RT, Grantham JJ, Higashihara E, Perrone RD, Krasa HB, Ouyang J, Czerwiec FS: Tolvaptan in patients with autosomal dominant polycystic kidney disease. N Engl J Med 2012;367:2407-2418

24 Cryer PE, Axelrod L, Grossman AB, Heller SR, Montori VM, Seaquist ER, Service FJ; Endocrine Society: Evaluation and management of adult hypoglycemic disorders: an endocrine society clinical practice guideline. J Clin Endocrinol Metab 2009;94:709-728.

25 Lalau JD, Race JM: Lactic acidosis in metformin-treated patients. Prognostic value of arterial lactate levels and plasma metformin concentrations. Drug Safety 1999;20:377384.

26 Rachmani R, Slavachevski I, Levi Z, Zadok B, Kedar Y, Ravid M: Metformin in patients with type 2 diabetes mellitus: reconsideration of traditional contraindications. Eur J Intern Med 2002;13:428.

27 Langone AJ, Chan L, Bolin P, Cooper M: Enteric-coated mycophenolate sodium versus mycophenolate mofetil in renal transplant recipients experiencing gastrointestinal intolerance: a multicenter, double-blind, randomized study. Transplantation 2011;91:470-478.

-28 Walz G, Budde K, Mannaa M, Nürnberger J, Wanner C, Sommerer C, Kunzendorf U, Banas B, Hörl WH, Obermüller N, Arns W, Pavenstädt H, Gaedeke J, Büchert M, May C, Gschaidmeier H, Kramer S, Eckardt KU: Everolimus in patients with autosomal dominant polycystic kidney disease. N Engl J Med 2010;363:830-840.

29 Tesar V, Ciechanowski K, Pei Y, Barash I, Shannon M, Li R, Williams JH, Levisetti M, Arkin S, Serra A: Bosutinib versus placebo for autosomal dominant polycystic kidney disease. J Am Soc Nephrol 2017;28:34043413.

30 US Food and Drug Administration: Qualification of Biomarker; Total Kidney Volume in Studies for Treatment of Autosomal Dominant Polycystic Kidney Disease, 2016.

31 Torres VE, Chapman AB, Devuyst O, Gansevoort RT, Perrone RD, Koch G, Ouyang J, McQuade RD, Blais JD, Czerwiec FS, Sergeyeva O: Tolvaptan in later-stage autosomal dominant polycystic kidney disease. N Engl J Med 2017;377:1930-1942. 